\title{
Management of acoustic neuroma
}

\section{A Wright, R Bradford}

The past 10 years have seen remarkable advances in the diagnosis and management of tumours that grow in the narrow space between the brain stem and cerebellum and the inside surface of the temporal bone-the so called cerebellopontine angle. The tumours that grow here are not uncommon, and the published annual incidence of symptomatic acoustic neuromas diagnosed during life is usually taken as

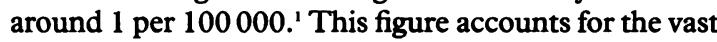
majority of all the different types of tumour found in this region. Most of these tumours are benign, but are located in a surgically awkward spot with important neurological structures close by.

Nowadays there is probably no good excuse to miss

Royal National Throat, Nose and Ear Hospital, London WC1X 8EE A Wright, professor of otolaryngology

Royal Free Hospital Medical School, London NW3 20 G

R Bradford, consultant

neurosurgeon

Correspondence to: Professor Wright.

BMF 1995;311:1141-4 the early diagnosis of tumours in this region, and this is important since if treatment by surgery or radiotherapy is contemplated then the smaller the tumour the better the results. ${ }^{2}$

\section{Contents of the cerebellopontine angle}

Running across the cerebellopontine angle from the brain stem are the facial (VII) and acoustic and vestibular (VIII) nerves on their way to the internal auditory meatus and thence to the muscles of facial expression and the cochlear and vestibular labyrinths respectively. At the apex of the cerebellopontine is the trigeminal $(V)$ nerve carrying sensation from the face

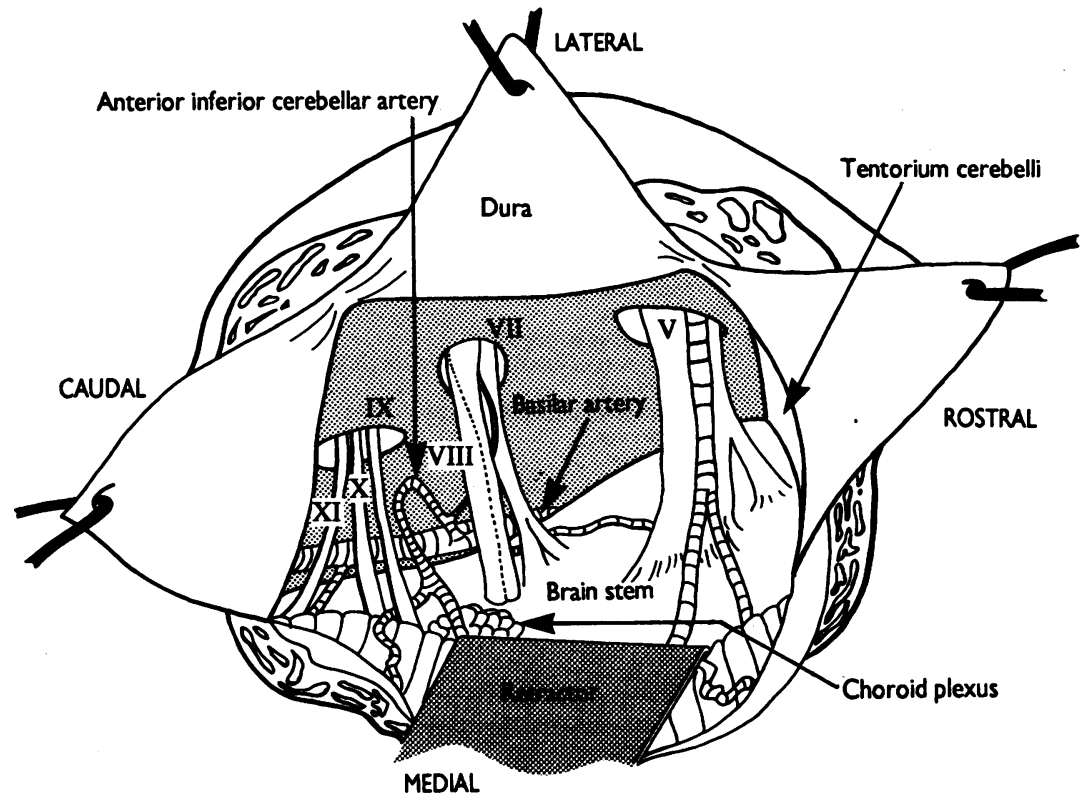

FIG 1-Left cerebellopontine angle through posterior craniotomy when patient is lying on right side in "park bench" position. In reality this much cerebellar retraction would not be used. The incised dura is held open by stay sutures. The lower cranial nerves pass from brain stem to their foramina in the temporal bone. The anatomy, especially of the vasculature, varies enormously. V=trigeminal nerve, VII=facial nerve, VIII= acousticovestibular nerve, $I X=$ glossopharyngeal nerve, $X=v a g u s$ nerve, $X I=$ spinal acessory nerve

Summary points
- The onset of unilateral auditory symptoms
requires investigation
- A unilateral sensorineural loss or tinnitus
when the eardrum is normal also needs further
investigation
- The definitive investigation is gadolinium
enhanced magnetic resonance scanning
- Neurological symptoms suggestive of com-
pression of the lower cranial nerves, ataxia, or
raised intracranial pressure may be caused by
benign tumours in the cerebellopontine angle
- Treatments include expectant care with
repeat scanning to assess tumour growth in
elderly people, and surgery with or without
stereotactic radiotherapy
- The smaller the tumour the lower the risks of
treatment

and supplying the muscles of mastication. At the base of the cerebellopontine angle are the lower cranial nerves (IX, X, and XI) on their way out of the skull through the jugular foramen to supply a wide range of functions but most importantly, from our point of view, to control swallowing, the airway, and the trapezius and sternomastoid muscles. Tongue movement is controlled by the hypoglossal (XII) nerve, which leaves a little lower down. Coursing through the cerebellopontine angle is the anterior inferior cerebellar artery, which is, usually, a branch of the basilar artery and supplies the underside of the cerebellum and parts of the brain stem, as well as having a labyrinthine branch that is the only supply to the inner ear (fig 1).

The symptoms that arise from tumours in the cerebellopontine angle are those that come from direct involvement or compression of the nerves themselves; compression of the brain stem, or in the latter stages from obstruction to the flow of cerebrospinal fluid produces an obstructive hydrocephalus and subsequent raised intracranial pressure.

\section{Tumours of the cerebellopontine angle}

Acoustic neuromas are both doubly misnamed and the most common tumours in this site. Not only do they most frequently occur on the superior portion of the vestibular nerve but they are also tumours of the nerve sheath rather than the nerve itself and so should really be called vestibular schwannomas (after Theodore Schwann, who described nerve sheaths 150 


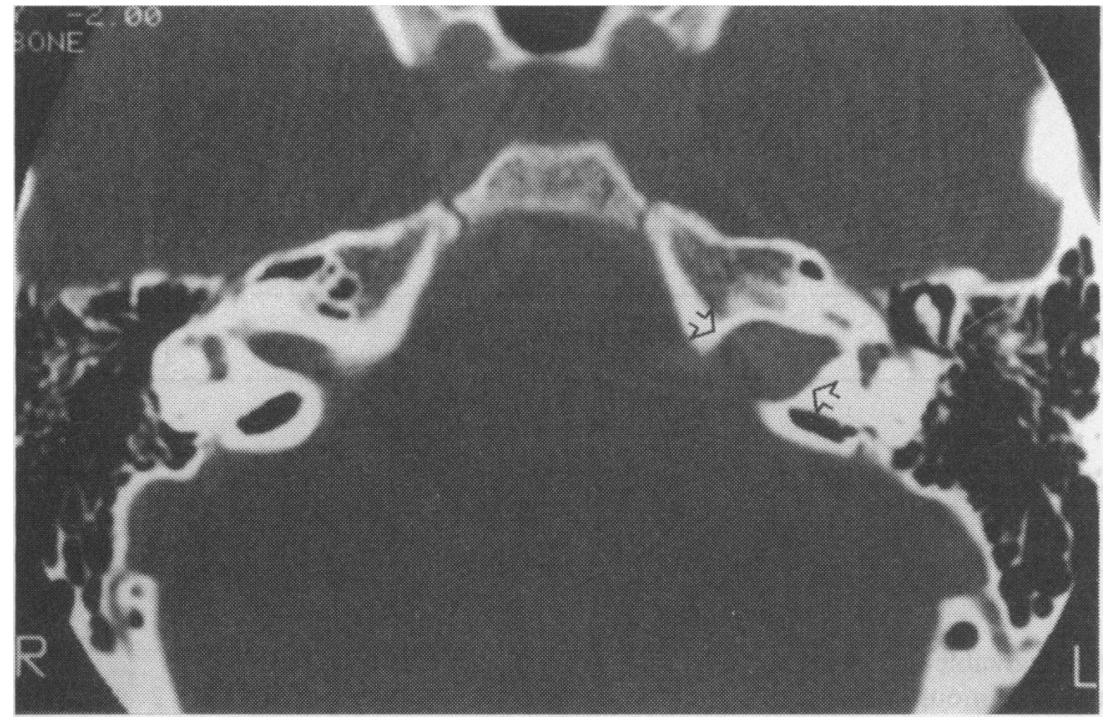

FIG 2-Axial computed tomogram taken before introduction of magnetic resonance imaging. The lefi internal auditory meatus is considerably widened (arrows) compared with the normal meatus on the right. Despite enhancement the almost spherical tumour in the posterior fossa is difficult to see, and a good evaluation of the size of the tumour and its attachment to other structures is impossible without more invasive investigations such as angiography

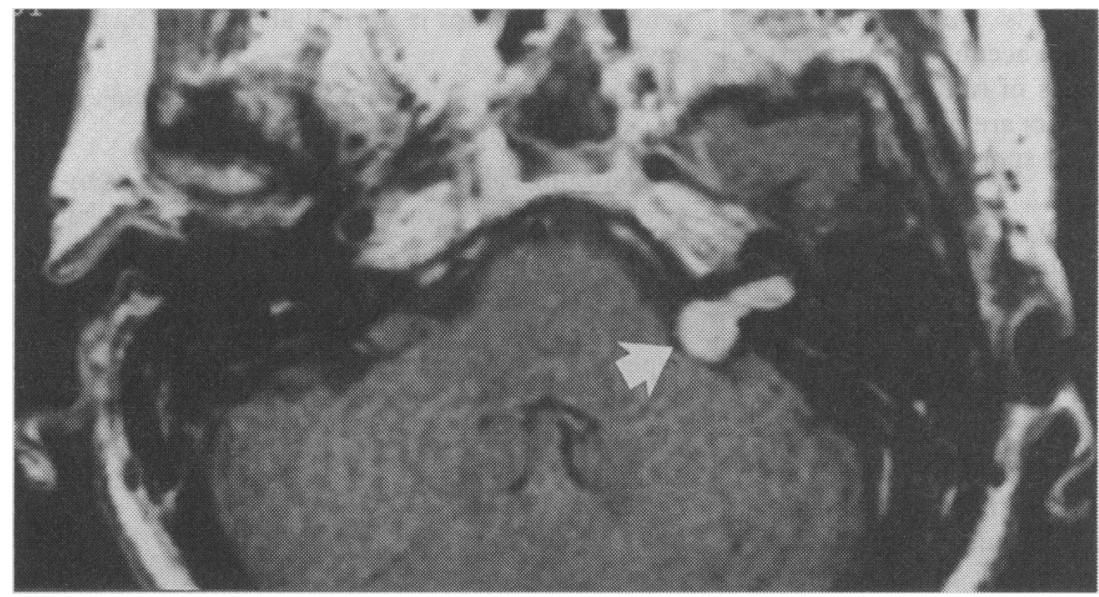

FG 3-Early magnetic resonance scan enhanced by gadolinium showing typical medium sized acoustic neurome (arrow) causing tinnitus and hearing loss of $50 \mathrm{~dB}$ (such enhancement of magnetic resonance imaging enables diagnosis of even small neuromas). The neuroma extends from the depths of the meatus and just indents the brain stem years ago). True acoustic neuromas form part of a familial syndrome called neurofibromatosis 2 , which consists of multiple cranial nerve tumours, along with neuromas on the spinal nerve roots which can cause spinal cord compression. Neurofibromatosis 2 is usually inherited as an autosomal dominant disorder and is due to a lesion on chromosome $22 \mathrm{q} 1 .^{3}$

The approximate incidences of the various tumours that can be found in the cerebellopontine angle are listed in table I.

\section{Symptoms}

Patients with acoustic neuromas seem to fall into two groups: those who present with relatively minor otolaryngological symptoms and those who present with more major neurological ones. This is probably related to the site of origin of the tumour along the acousticovestibular nerve bundle. Those that arise in the internal auditory meatus rapidly compress the auditory nerve and quickly give some combination of alteration in hearing (such as distortion and not necessarily hearing loss), tinnitus, and a feeling of pressure in the ear on the affected side. The tumours that arise from the nerve in the cerebellopontine angle itself may have no or minimal symptoms until the brain stem is compressed with the onset of noticeable clumsiness (ataxia) and then as the intracranial pressure rises headache, vertigo, vomiting, fever, and even visual changes as papilloedema occurs (tables II-IV).

The important thing to do is to take bilateral symptoms very seriously, especially if they are pro- gressive or do not respond to standard treatment. A common failing is to assume that a feeling of pressure and muffled hearing is due to obstruction of the eustachian tube, even though the eardrum is normal. Similarly a classic trigeminal neuralgia that fails to respond or responds erratically to carbamazepine is frequently caused by irritation to the trigeminal nerve by a tumour in the cerebellopontine angle.

When in doubt investigate.

\section{Diagnosis}

As always, the diagnosis of a tumour in the cerebellopontine angle is made on the basis of the history and examination and a suspicion that these two warrant further investigation. With small acoustic neuromas the results of examination are usually absolutely normal as may be the results of tuning fork tests since any initial hearing loss is nearly always high frequency.

If pure tone audiometry is available then this should be performed, and provided that the bone conduction thresholds are reliable then any asymmetry of the audiogram in the absence of a known earlier cause of hearing loss (such as meningitis, head injury, previous ear surgery, etc) should be considered clinically significant.

We think that should a referral to an ear, nose, and throat surgeon be considered at this stage then the referral letter might usefully contain words to the effect that: "it would be appreciated if an acoustic neuroma be excluded."

If any neurological signs exist (tables V and VI) then there are two options. If the facilities exist then the next step is referral by the general practitioner for investigative imaging. This avoids the double delay of the patient awaiting both a hospital outpatient and then a scan appointment if the scan is positive, while a negative initial scan is an immediate source of relief to both the patient and the doctor.

At present the definitive examination is a gadolinium enhanced magnetic resonance scan (fig 3 ). This can detect lesions of $2 \mathrm{~mm}$ in diameter and probably smaller. An alternative which should be cheaper and nearly as reliable, is the fast spin echo technique applied to magnetic resonance scanning which does

TABLE I-Prevalence of tumours in cerebellopontine angle

\begin{tabular}{lc}
\hline Tumour & $\begin{array}{c}\text { Prevalence } \\
(\%)\end{array}$ \\
\hline Acoustic neuroma & 75 \\
Meningioma & 6 \\
Cholesteatoma & 6 \\
$\quad$ Epidermoid cyst & \\
Arising from temporal bone & 3 \\
Glioma & 10 \\
Other types & \\
$\quad$ Metastatic tumours & \\
Osteomas & \\
Osteogenic sarcomas & \\
$\quad$ Anguromas of trigeminal, facial, or glossopharyngeal nerve & \\
Papillomas of choroid plexus & \\
$\quad$ Teratomas & \\
$\quad$ Lipomas & \\
\hline
\end{tabular}

TABLE I-Symptoms of acoustic neuromas presenting to ear, nose, and throat surgeons. Values are percentagest

\begin{tabular}{lcc}
\hline Symptom & $\begin{array}{c}\text { Primary } \\
\text { complaint }\end{array}$ & $\begin{array}{c}\text { Secondary } \\
\text { complaint }\end{array}$ \\
\hline Alterations in hearing (distortion, hearing loss, & & \\
$\quad$ tinnitus) & 60 & 16 \\
Headache & 16 & 15 \\
Unsteadiness & 7 & 30 \\
Fifth nerve symptoms & 7 & 15 \\
Earache & 4 & 4 \\
Vertigo & 3 & 3 \\
Sudden profound loss of hearing & $\mathrm{NK}$ & $\mathrm{NK}$ \\
\hline
\end{tabular}

NK= Not known 
TABLE II-Symptoms that can arise from compression of nearby structures

\begin{tabular}{lc}
\hline Symptom & Structure \\
\hline $\begin{array}{l}\text { Atypical trigeminal } \\
\text { neuralgia }\end{array}$ & $\mathrm{V}$ \\
$\begin{array}{c}\text { Tic douloureux } \\
\text { Progressive painless } \\
\text { facial weakness }\end{array}$ & $\mathrm{VII}$ \\
$\begin{array}{c}\text { Hearing loss and/or } \\
\text { tinnitus in other ear }\end{array}$ & Brain stem \\
Hoarse weak voice & $\mathrm{X}$ \\
\hline
\end{tabular}

TABLE IV-Symptoms that might present as neurological problems as brain stem compression occurs and raised intracranial pressure develops

Symptom

Clumsiness, poor balance

Headache

Vertigo

Vomiting

Fevers

Deterioration in mental state

Visual changes

Fits

TABLE V-Neurological signs sometimes associated with acoustic neuromas

Neurological sign

\section{Spontaneous nystagmus}

Loss or reduction of corneal reflex

Reduced hearing in whisper tests

Weber test lateralises to "good ear"

Wercer

not require gadolinium but does require more sophisticated software for the scanner (figs 4-6). A normal fast spin echo scan excludes a tumour while an abnormal one may require gadolinium for confirmation and sizing of the growth.

If magnetic resonance imaging is not available then enhanced computed tomography will detect most tumours with an intracranial dimension of $1.0 \mathrm{~cm}$ or more (fig 2). The scan must be enhanced since many acoustic neuromas have the same scan density as surrounding brain and are therefore not detectable in non-enhanced procedures. Widening of the internal auditory meatus by an acoustic neuroma can also be seen on a computed tomogram and this, in itself, is valuable in deciding whether, in an otherwise equivocal case, to go ahead with magnetic resonance imaging.

\section{Management}

The decision as to what is to be done for a patient with an acoustic neuroma is a complex interplay of the needs and expectations of the patient, the size of the tumour, and the local expertise.

Acoustic neuromas grow, although the rate of increase cannot yet be determined until repeat scans are performed after a suitable delay. However, as a guiding principle the rate of growth seems to be slower in elderly people, and postmortem studies have shown an incidence of acoustic neuromas which have not contributed to the death of the person of between $0.8 \%{ }^{5}$ and $2.7 \%{ }^{6}$ Nevertheless, in younger people it is almost certain that most tumours will continue to grow and eventually cause clinically significant problems, then death if not treated.

Surgery, however, has its risks and those risks increase with the size of the tumour but decrease with experience of the operating team.

TABLE VI-Typical features associated with peripheral nystagmus arising from the inner ear or vestibular nerve contrasted with those arising centrally from the brain stem or higher ${ }^{\star}$

\begin{tabular}{l|l}
\hline Peripheral nystagmus & Central nystagmus \\
\hline $\begin{array}{l}\text { Fast phase in same direction } \\
\text { whatever the direction of gaze }\end{array}$ & $\begin{array}{l}\text { Usually direction changing } \\
\text { Often vertical } \\
\text { Horizontal or rotary } \\
\begin{array}{l}\text { Never vertical } \\
\text { Decreasing severity over two weeks }\end{array} \\
\text { two weeks } \\
\text { May be bizarre }\end{array}$ \\
\hline
\end{tabular}

*Nystagmus is defined here as an involuntary, rhythmic oscillation of both eyes in phase together-that is, conjugate eye movements. Nystagmus both eyes in phase together-that is, conjugate eye movements. Nystagmus
usually has a fast phase and a slow phase. The slow phase is the abnormal usually has a fast phase and a slow phase. The slow pha
movement whilst the fast phase is a corrective saccade.

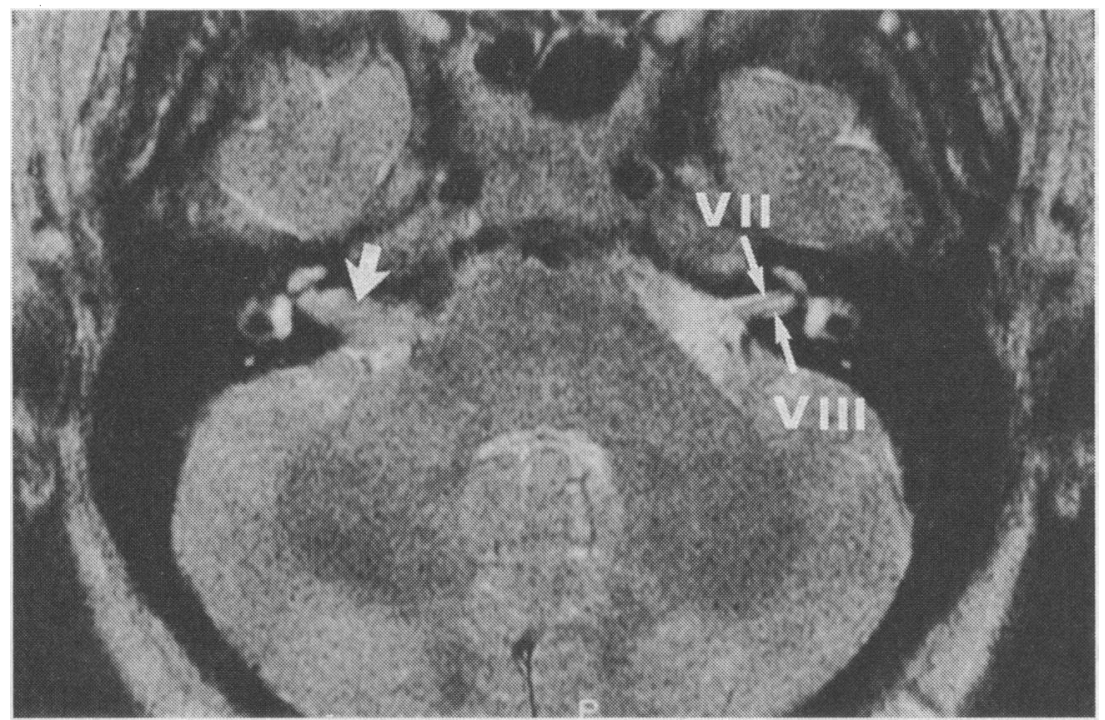

FIG 4-Magnetic resonance scan from scanner with new processing software that enhances image without need for gadolinium (T2 weighted fast spin echo technique) and gives a clear view of structures in the posterior fossa. Facial (VII) and acousticovestibular (VIII) nerves on the left are normal, and the heavy arrow shows a solid tumour in the internal meatus on the right

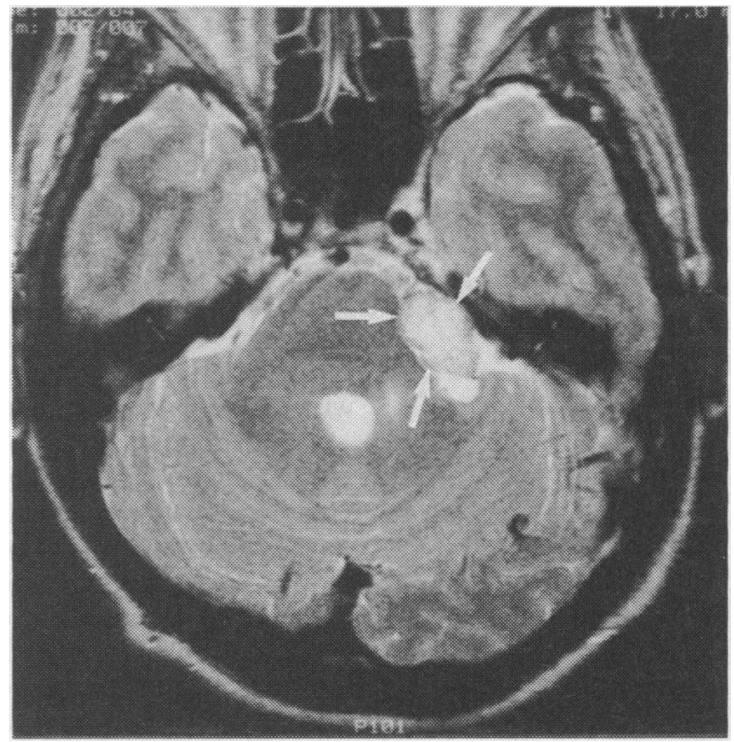

FIG 5-Magnetic resonance scan showing large left sided mass (arrows) in cerebellopontine angle but without any extension into internal auditory meatus in patient presenting with right sided tinnitus. In this case the lack of rigorous labelling on the original scans caused some early diagnostic confusion. However, the brain stem is quite distorted, and at surgery a trigeminal neuroma was found

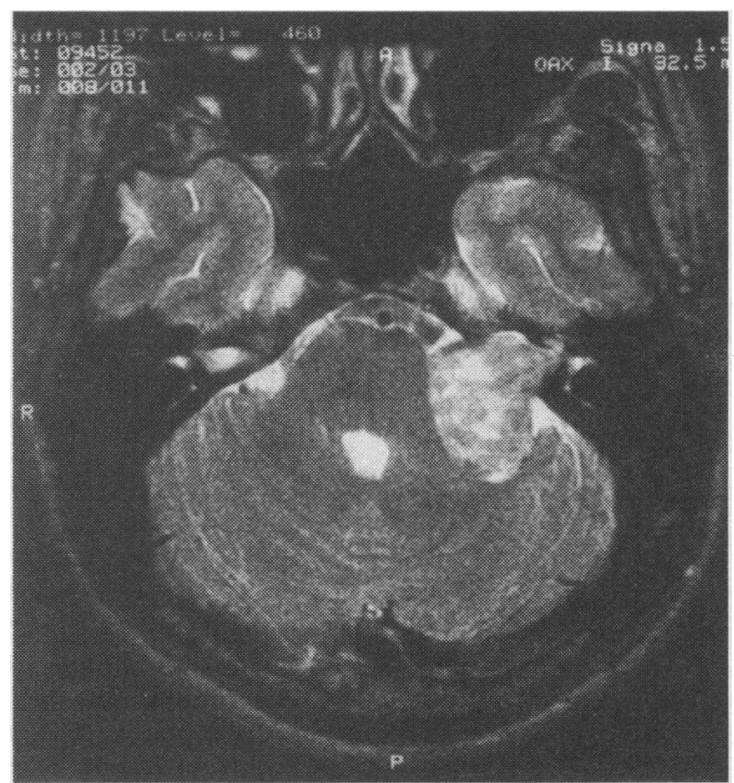

FIG 6-Magnetic resonance scan of 30 year old man who presented with symptoms and signs of brain stem compression and rising intracranial pressure. He had not apparently noticed or been bothered about his slow loss of hearing. Function of the facial nerve was normal preoperatively

Unless the tumour is small or medium sized, then the chances of preserving useful hearing are slight. Sudden profound hearing loss from surgery seems to have a far greater psychological and social impact than the same loss which has occurred slowly from tumour growth. The best way of preserving hearing is not to remove the tumour but this requires conscientious follow up with repeat scans and even then there is an incidence of spontaneous severe sensorineural hearing loss with these tumours.

The facial nerve runs across the surface of an acoustic neuroma and as the tumour increases in size the nerve becomes stretched and thins out so that it may become almost web-like around one side of the tumour. Dissecting the tumour from the nerve is difficult, and even if anatomical continuity is maintained the nerve may not function fully or at all postoperatively. A facial palsy is an awful deformity to inflict on someone when it is unlikely to be caused by the tumour itself. Careful discussion about this aspect of the surgery is, we think, mandatory. Studies have 
shown that if small fragments of tumour capsule are left behind at surgery then $50 \%$ of them do not continue to grow. Those that do regrow can in due course be removed, debulked, or treated with stereotactic radiotherapy (see later).

Although acoustic neuromas grow on the vestibular nerve, there is frequently some balance function left and so removal of the tumour may cause vertigo. This usually settles to be replaced by unsteadiness, which resolves as central compensation occurs. This compensation is less good in elderly people, who may be left with permanent unsteadiness.

If the tumour is large and compressing the brain stem damage can occur during removal and cause many major neurological symptoms and signs. Long tract symptoms with major paralysis on the same side of the body, central type nystagmus, and diplopia (damage to IV and VI) are all possible. The lower cranial nerves can be affected with dysphagia and laryngeal incompetence so that a tracheostomy and feeding through a nasogastric tube or gastrostomy are needed. Damage to the anterior inferior cerebellar artery can also cause brain stem and cerebellar infarction with gross ataxia that is very slow, or occasionally fails, to settle.

The catalogue of surgical complications is depressing and makes early diagnosis all the more important.

The surgical approaches and techniques that are available fall into three main groups.

The middle cranial fossa approach-For small tumours that lie in the internal auditory meatus and with residual hearing, this approach has a low morbidity and offers an extremely good chance of preserving the hearing with what should be a negligible risk to the facial nerve.

The transmastoid, translabyrinthine (translab) approach-This approach by way of an extended mastoidectomy has a low morbidity when carefully performed but destroys the hearing. It is suitable for all sized tumours, although possibly extremely large acoustic neuromas are better approached by a posterior cranial fossa route.

Posterior cranial fossa (retrosigmoid) approach-This is suitable for all but extremely small tumours and offers the choice of preserving the hearing in patients with small to medium sized tumours. However, the incision through the neck muscles can cause long term postoperative discomfort, and retraction of the cerebellum, to gain access to the cerebellopontine angle, may occasionally cause marked persistent ataxia. For large tumours, however, and especially for tumours that are not acoustic neuromas, this is probably the safest route of approach.

We think that any unit offering surgery for tumours of the cerebellopontine angle should have available, as a minimum, intraoperative facial nerve monitoring, an ultrasonic surgical aspirator, and intensive care facilities for the immediate postoperative period because of the risk of bleeding into the posterior cranial fossa. Surgical teams should be able to offer the full variety of approaches so that the individual patient can have the most suitable plan of treatment. This requires the cooperation of ear, nose, and throat surgeons, neurosurgeons, and anaesthetists, which in effect means that work will need to be based in appropriate centres.

\section{Stereotactic radiotherapy}

Advances in focusing multiple beams of radiotherapy and the use of high energy gamma radiation has allowed the development of stereotactic techniques which, when correctly used, can deliver highly localised levels of radiation with minimal damage to surrounding structures. ${ }^{7}$ Because of the morbidity of surgical techniques, especially to the facial nerve and to hearing, there is a move towards using stereotactic radiotherapy for tumours with the maximum dimension of less than $3 \mathrm{~cm}^{8}$ Whether the use of such treatment will serve only to delay inevitable surgery remains to be seen, but it may become a valuable form of treatment for elderly people and for those with bilateral acoustic neuromas for whom the preservation of hearing is extremely important.

\section{Combined surgery and radiotherapy}

The published work on stereotactic radiotherapy seems to show that it is of most benefit for those with small tumours. Therefore surgery to debulk a large tumour which has a low operative morbidity compared with attempted total removal, combined with postoperative stereotactic radiotherapy may become a useful combination, ${ }^{9}$ although the long term value of this form of treatment is yet to be decided.

\section{The future}

Advances in imaging technology have made the detection of small tumours much easier, and this in itself has posed problems as to what to do with the relatively asymptomatic growths in young people. To be able to reliably predict the rate of growth of an individual tumour would be a great advantage, and work is in progress to identify specific tumour markers that may be helpful. These could be obtained endoscopically through small posterior cranial fossa burr holes. It may be that precision radiotherapy will then be able to prevent the further growth of these small tumours and that surgery is relegated to the treatment of those tumours that have escaped early detection or fail to respond to radiotherapy.

At present, however, one great benefit would be the ability to reliably preserve residual hearing during surgery. Intraoperative monitoring of the electrical function of the cochlea and auditory brain stem is possible (although difficult) and is practised by some groups. ${ }^{10}$ Whether widespread acceptance of this technique occurs will depend on the usefulness of the residual hearing after such surgery, and there is much debate as to what constitutes useful residual hearing, ${ }^{11}$ but in those few people with absent or poor hearing in the other ear such techniques may become mandatory. The development of brain stem auditory implants, whereby electrodes are placed on the brain stem cochlear nuclei (which conveniently lie on the surface of the brain stem), might mean that even though the cochlear nerve is damaged "hearing" can be preserved.

1 Tos M, Thomsen J, Charabi S. Epidemiology of acoustic neuromas: has the incidence increased during the last years? In: Tos $M$, Thomsen $J$, eds. Proceedings of the first international conference on acoustic neuroma, Copenhagen August 1991. New York: Kugler, 1992.

2 Ramsden RT, Moffat DA. Intracanalicular acoustic neuromas: the case for early surgery. Clin Orolaryngol 1994;19:1-2.

3 Troffater JA, MacCollin MM, Rutter J. A novel Moesin-Ezrin-Radixin-like gene is a candidate for the neurofibromatosis type 2 tumor suppressor. Cell 1993;72:791-800.

4 Morrison AW. Management of sensorineural deafness. London: Butterworth, 1975:52.

5 Leonard JR, Talbot ML. Asymptomatic acoustic neurilemmoma. Archives of Otolaryngology 1970:91:117-24.

6 Hardy M, Crowe SJ. Early asymptomatic acoustic tumour. Arch Surg 1936;32:292-306.

7 Ogunrinde OK, Lunsford LD, Flickinger JC, Konziolka DS. Cranial nerve presentation after stereotactic radiotherapy for small acoustic tumors. Anch Neurol 1995;52:73-9.

8 Pollock BE, Lunsford LD, Kondziolka D, Flickinger JC, Bissonette DJ, Kelsey SF, et al. Outcome analysis of acoustic neuroma management: comparison of microsurgery and stereotactic radiosurgery. Neurosurgery 1995;36:224-9.

9 Lunsford LD, Linksey ME. Stereotactic treatment of patients with acoustic tumors. Otolaryngol Clin North Am 1992;25:471-91.

10 Silverstein H, McDaniel A, Norrell H, Haberkamp T. Hearing preservation after acoustic neuroma surgery with intraoperative direct eighth cranial after acoustic neuroma surgery with intraoperative direct eigh
nerve monitoring. Otolanyngol Head Neck Surg 1986;95:285-91.

11 Brookes GB, Woo J. Hearing preservation in acoustic neuroma surgery. Clin Otolaryngol 1994;19:204-14. 